\title{
Effects of Palivizumab Guideline Changes on RSV Admissions in Patients with Congenital Heart Disease and Prematurity
}

\author{
John Pham¹, Stephen Nageotte², Jon Detterich², Grace Kung² \\ ${ }^{1}$ Department of Pediatrics, Children's Hospital Los Angeles, Los Angeles, CA, United States of America \\ ${ }^{2}$ Division of Cardiology, Department of Pediatrics, Children's Hospital Los Angeles, Los Angeles, CA, United States of America \\ Email: johnphamMD@gmail.com
}

How to cite this paper: Pham, J., Nageotte, S., Detterich, J. and Kung, G. (2021) Effects of Palivizumab Guideline Changes on RSV Admissions in Patients with Congenital Heart Disease and Prematurity. World Journal of Cardiovascular Diseases, 11, 34-44. https://doi.org/10.4236/wjcd.2021.111005

Received: December 14, 2020

Accepted: January 18, 2021

Published: January 21, 2021

Copyright $\odot 2021$ by author(s) and Scientific Research Publishing Inc. This work is licensed under the Creative Commons Attribution International License (CC BY 4.0).

http://creativecommons.org/licenses/by/4.0/

\begin{abstract}
Background: Respiratory syncytial virus (RSV) causes significant morbidity and mortality in patients with a history of prematurity and congenital heart disease (CHD). In 2014, the guidelines for Palivizumab became more restrictive for this population. We hypothesized the percentage of RSV+ admissions would increase overall and in this target group (TG) specifically. Methods: We conducted a retrospective review of patients under age 2 years admitted with bronchiolitis two seasons prior to the change (Pre) and two seasons after (Post). Our TG included patients who were eligible prior to the 2014 changes but currently no longer eligible. We used chi-square analysis to answer the two main hypotheses: 1) Percent RSV+/total bronchiolitis Pre vs Post and 2) Percent of TG/RSV+ Pre vs Post. Results: 1283 patients (546 pre, 737 post) were admitted with the diagnosis of RSV between 2012-2016, 866 actually tested positive for RSV (367 Pre, 499 Post). There was no significant difference in the number of total patients admitted with RSV (Pre $=67.2 \%$, Post $=$ $67.7 \%$ ) or in our TG (Pre 7.1\% vs Post $8.2 \%$ ). TG overall had a more complicated course: longer length of stay, median 5 days, IQR 2 - 12 vs 3 days, IQR 1 - 5, ( $<<0.001)$, intensive care unit admissions ( $36 \%$ vs $22.8 \%, p=0.02)$, positive pressure ventilation $(25.4 \%$ vs $15.4 \%, \mathrm{p}=0.03)$ and intubation $(16.4 \%$ vs $6.8 \%, \mathrm{p}=0.004)$, but there was no difference Pre vs Post. Conclusion: The TG had an overall higher acuity, but there was no increase in the number of patients hospitalized with RSV or severity as a result of the Palivizumab guideline changes.
\end{abstract}

\section{Keywords}

2014, American Academy of Pediatrics, Children, Congenital Heart Disease, Guidelines, Infants, Palivizumab, Prematurity, Respiratory Syncytial Virus 


\section{Introduction}

Respiratory syncytial virus (RSV) accounts for 2.1 million outpatient visits and 57,527 hospitalizations among children less than 5 years old (yo) in the United States, thus making it the most common cause of lower respiratory tract infections among this population. While RSV is known to cause croup and pneumonia, it is recognized as the most common cause of bronchiolitis, particularly in children less than 1 year of age [1]. Transmission of this virus occurs through oral and nasal secretions. Studies have shown this common virus is associated with increased morbidity and mortality in patients with a history of prematurity and congenital heart disease (CHD) [1].

In 1998, a humanized monoclonal antibody against RSV, Palivizumab, was licensed by the Food and Drug Administration for patients at highest risk as described above. Palivizumab is given monthly with a maximum of 5 doses during RSV season, which spans from November to April, with a peak in January and February [2]. Since its availability, the American Academy of Pediatrics (AAP) has released and updated their recommendations on the use of Palivizumab 5 times with the most recent change in 2014, just 2 years after their prior update [3]. These updates have been made based on ongoing assessments in various areas which include: seasonality of RSV circulation [4] [5], pharmacokinetics of Palivizumab [6], risk stratification with other comorbidities (e.g. Down syndrome, cystic fibrosis) [7] [8], mortality rates of hospitalized children with RSV [9], declining incidence of bronchiolitis hospitalizations [10], and cost analysis reports [11]. However, there is limited information on the impact of individual guidelines changes on these outcomes.

According to the recommendations published in 2012, children who met one of the following criteria were eligible for monthly Palivizumab prophylaxis: 1) Gestational age (GA) $<32$ weeks (wks) and $<12$ months old (mo) at the beginning of RSV season; 2) GA $32-35$ wks and $<3$ mo at the start of the RSV season or born during the RSV season and have an increased risk of exposure to RSV (either attends child care, have another child $<5$ yo in the same household, or both); 3) Age < 24 mo with hemodynamically significant CHD, chronic lung disease, congenital airway abnormalities, or neuromuscular disease; 4) Immunocompromised children $<24$ mo.

The 2014 update, however, became more restrictive as the AAP redefined their definition of "high-risk". Most notably with this significant change, infants $<12$ mo at the start of RSV season who are born between $29-32$ wks GA and children age 1 - 2 yo with underlying high-risk medical conditions (hemodynamically significant $\mathrm{CHD}$, chronic lung disease, congenital airway abnormalities, and neuromuscular disease) are no longer recommended for RSV prophylaxis. Babies 32 - 35 wks GA who are $<3$ mo at the start of RSV season or born during RSV season are no longer eligible for Palivizumab as well [3].

The aims of this study were: 1) To evaluate the effects of the 2014 guideline changes on the number of patients admitted for bronchiolitis who tested positive 
for RSV (RSV+); and 2) The number of RSV+ patients in the target population for whom the guidelines changed. We hypothesized that the new guidelines would result in an increase in RSV+ admissions overall (aim 1) and in the target group (aim 2).

\section{Methods}

\subsection{Study Design}

A retrospective chart review was performed at a tertiary care center in the Southwestern United States. We obtained institutional review board approval for this cross-sectional study on patients admitted for bronchiolitis between 2012 and 2016. Diagnosis Related Group code 138 and International Classification of Disease, Ninth Revision (ICD-9: 079.6, 466.11, 480.1) and Tenth Revision (ICD-10: B97.4, J12.1, J21.0) codes were used to identify patients who were diagnosed with bronchiolitis or RSV bronchiolitis during their hospitalization. RSV infection was confirmed through multiplex polymerase chain reaction (PCR) assays. The primary method for detection of RSV was by viral FilmArray Respiratory Panel Torch Systems from BioFire Diagnostics (Salt Lake City, Utah), which detects 17 of the most common respiratory viral pathogens.

\subsection{Variables}

Pertinent demographic and clinical characteristics were obtained through our electronic medical record system and manually reviewed. Information obtained included: gender, age during admission, GA, confirmation of RSV, and history of CHD. In addition, we did a subanalysis of severity of disease during admission: length of stay (LOS), intensive care unit (ICU) admission, use of oxygen $(\mathrm{O} 2)$, positive pressure ventilation (PPV), and need for intubation.

\subsection{Inclusion Criteria}

Palivizumab eligibility for patients admitted during the 2012-2014 RSV season was based on the 2012 AAP recommendations. The most recent 2014 guidelines were applied to patients admitted after the change (2014-2016). We included patients under 2 yo who were admitted with the above diagnostic codes from timeline of November 1 through March 31 two years prior to the change in guidelines (2012-2014), designated "Pre", and two years after (2014-2016), designated "Post". We included patients admitted for a clinical diagnosis of bronchiolitis with and without viral panel testing. We recorded whether patients had viral testing and tested both RSV+/all bronchiolitis admissions as well as RSV+/bronchiolitis admissions with viral testing only; but the final analysis included all patients with a clinical diagnosis. We defined our target group (TG) to include patients specifically affected by the 2014 guideline changes. Target Group 1 (TG-1) consisted of patients with hemodynamically significant CHD between $1-2$ yo who were no longer eligible for Palivizumab. Target Group 2 (TG-2) were patients born at 29 - 35 wks GA and were less than 1 year of age during time of admis- 
sion and were no longer eligible. Those remaining who did not meet the criteria for the target population were categorized into our control group (CG) for whom the guidelines would not have changed.

\subsection{Congenital Heart Disease}

CHD was defined as infants with any of the following: 1) cyanotic congenital defects; 2) acyanotic heart disease who are receiving medications to control congestive heart failure until they receive cardiac surgical repair; 3) moderate to severe pulmonary hypertension. Per the AAP recommendations, the following patients did not classify under our definition of CHD and thus were not included in our target population: hemodynamically insignificant heart disease (e.g. atrial and ventricular septal defects and patent ductus arteriosus not requiring medications for pulmonary overcirculation, mild coarctation of the aorta, uncomplicated aortic stenosis, and pulmonary stenosis), lesions adequately corrected by surgery (unless the patient is receiving medication for congestive heart failure), and patients with mild cardiomyopathy who are not receiving medical therapy for their condition [3].

\section{Analysis}

Chi-square test was used to evaluate associations between categorical variables. Fisher's exact test was applied when the analysis involved $\leq 10$ patients for any particular variable. Median ( $25^{\text {th }}$ to $75^{\text {th }} \mathrm{IQR}$ ) was used to describe LOS. Two-sample t-test was used for normally distributed continuous data and nonparametric analysis was used for data that was not normally distributed, defined by the Shapiro-Wilk test. A "p" value of $\leq 0.05$ was considered statistically significant. The power analysis for our first aim with a sample size of 1283 yielded $11 \%, 43 \%, 82 \%$, and $95 \%$ power to detect a difference in RSV+ percentages of $2 \%, 5 \%, 8 \%$ and $10 \%$ respectively; therefore, we were underpowered to detect a difference $<8 \%$ between groups. The power analysis for our second aim with a sample size of 866 yielded $9 \%, 75 \%$, and $84 \%$ power to detect a difference in RSV+ percentages of $2 \%, 9 \%$, and $10 \%$ respectively; therefore, we were underpowered to detect a difference $<10 \%$ between groups. All analyses were performed using JMP Pro 12 software (Copyright @ 2016 SAS Institute Inc.).

\section{Results}

Initially, 1297 children < 2 yo admitted with the diagnosis of bronchiolitis were identified by Diagnosis Related Group, ICD-9 and ICD-10 codes, 14 children were excluded due to insufficient data (gestational age was unknown), Figure 1. The remaining 1283 patients were categorized into a Pre group ( $\mathrm{n}=546$ admissions 2012-2014) and Post group ( $\mathrm{n}=737$ admissions 2014-2016). In the Pre group 434 patients (79.5\%) and in the Post group 594 patients (80.6\%) had viral testing. Of those, $367(67.2 \%)$ in the Pre group and 499 (67.7\%) in the Post group were RSV positive. There was no significant change in the number of RSV+ admissions Pre (367/546) vs Post (499/737), Table 1, $\mathrm{p}=0.85$. Limiting 


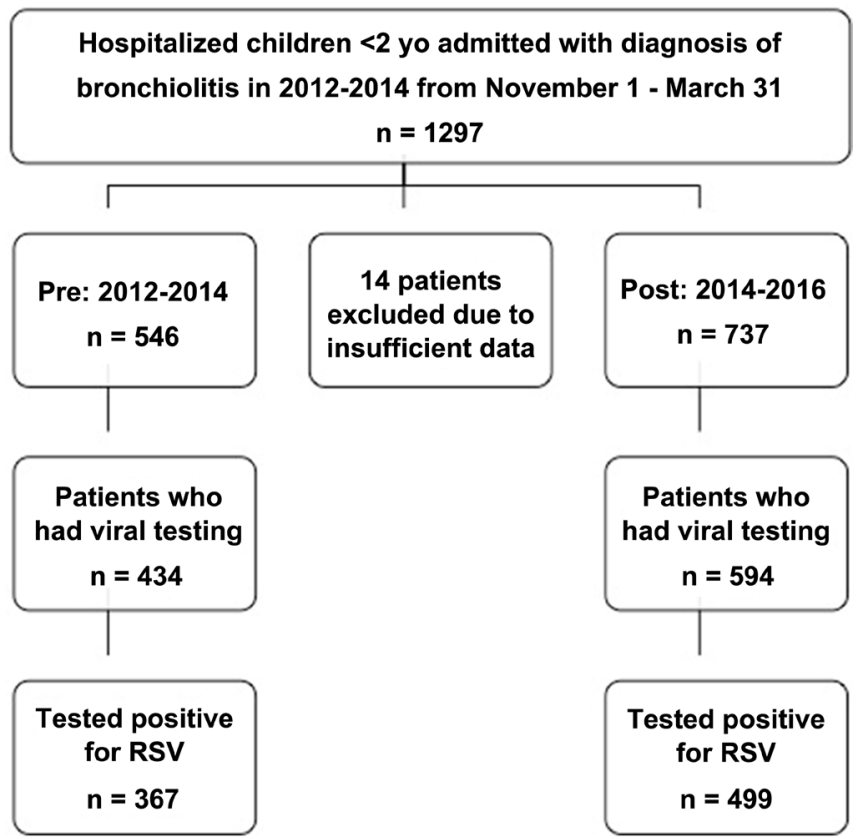

Figure 1. Patient group description. Abbreviations: RSV-Respiratory Syncytial Virus; yo-years old.

Table 1. Patient characteristics of all children ages 0 - 2 yo admitted with a diagnosis of bronchiolitis from 2012-2016; and markers of disease severity for the target group from 2012-2016.

\begin{tabular}{|c|c|c|c|c|c|}
\hline \multicolumn{6}{|c|}{ Demographics for all patients } \\
\hline & \multicolumn{2}{|c|}{ 2012-2014 } & \multicolumn{2}{|c|}{ 2014-2016 } & \multirow[b]{2}{*}{ p-value } \\
\hline & $\mathrm{n}($ total 546) & $\%$ & n (total 737) & $\%$ & \\
\hline Sex $($ Female $n=534)$ & 210 & $38.5 \%$ & 324 & $44 \%$ & 0.05 \\
\hline Had viral testing $(n=1028)$ & 434 & $79.5 \%$ & 594 & $80.6 \%$ & 0.08 \\
\hline$R S V+(n=866)$ & 367 & $67.2 \%$ & 499 & $67.7 \%$ & 0.85 \\
\hline Age $0-1$ yo $(n=693)$ & 299 & $81.5 \%$ & 394 & $79 \%$ & 0.40 \\
\hline Age $1-2$ yo $(n=173)$ & 68 & $18.5 \%$ & 105 & $21 \%$ & 0.40 \\
\hline Target Group $(n=67)$ & 26 & $7.1 \%$ & 41 & $8.2 \%$ & 0.54 \\
\hline 1) CHD 1 - 2 yo $(n=11)$ & 6 & $1.6 \%$ & 5 & $1 \%$ & 0.41 \\
\hline 2) $29-35$ wks $G A,<1$ yo $(n=56)$ & 20 & $5.4 \%$ & 36 & $7.2 \%$ & 0.30 \\
\hline \multicolumn{6}{|c|}{ Severity of Disease Variables for Target Group (TG) } \\
\hline & \multicolumn{2}{|c|}{$2012-2014$} & \multicolumn{2}{|c|}{ 2014-2016 } & p-value \\
\hline Median LOS (days) & 3 & $\begin{array}{c}\mathrm{IQR} \\
2-12\end{array}$ & 5 & $\begin{array}{c}\text { IQR } \\
3-11\end{array}$ & 0.30 \\
\hline ICU Admissions & 6 & $23.1 \%$ & 18 & $43.9 \%$ & 0.12 \\
\hline Supplemental Oxygen & 20 & $76.9 \%$ & 33 & $80.5 \%$ & 0.76 \\
\hline PPV & 5 & $19.2 \%$ & 12 & $29.3 \%$ & 0.40 \\
\hline Intubations & 5 & $19.2 \%$ & 6 & $14.6 \%$ & 0.74 \\
\hline
\end{tabular}

Abbreviations: CHD—Congenital Heart Disease; GA-Gestational Age; ICU-Intensive Care Unit; LOSLength of stay; PPV—Positive Pressure Ventilation; RSV—Respiratory Syncytial Virus; yo-years old. 
the denominator to only those who had viral testing had a similar negative result (data not shown).

\subsection{Target Population-Patients for Whom the Guidelines Changed}

In the patients for whom the guidelines changed, there were 26 patients (26/367 $=7.1 \%)$ of all proven RSV + admissions in the Pre group compared to 41 patients $(41 / 499=8.2 \%)$ in the Post group. There was no difference in the percentage of target group RSV+ admissions between the two eras $(\mathrm{p}=0.54)$.

During the overall study time period 2012-2016, the TG was sicker compared to the CG. The TG had a longer LOS, median 5 days (IQR 2 to 12) vs 3 days (IQR 1 to 5) for the CG ( $\mathrm{p}<0.001)$. TG had higher rate of ICU admission 36\% vs $22.8 \%(\mathrm{p}=0.02)$. There was a difference in use of PPV with $25.4 \%$ and $15.4 \%$ $(\mathrm{p}=0.03)$. There was also increased intubation compared to CG $16.4 \%$ vs $6.8 \%$ $(\mathrm{p}=0.004)$. There was no significant change in the use of supplemental $\mathrm{O} 2$ (Figure 2). However, disease severity among the TG did not worsen after the new guidelines were implemented (Table 1).

We then separated the TG into two sub-groups TG-1 (1 - 2 year olds with CHD) and TG-2 (29 - 35 wks GA less than 1 year of age). There were too few subjects in TG-1 to analyze. Therefore, TG-2 mirrored the findings of the overall TG. There was no increase in number of hospital admissions, however, there was an increase in the number of ICU admissions after the new guidelines were implemented (Table 2).

\subsection{Chronological Age at Admission}

We evaluated disease severity by age category (0 - 3 mo, 3 - 6 mo, 6 - 12 mo, and $>12 \mathrm{mo}$ ) for all children hospitalized with RSV during the overall study period and found increased disease severity among infants $0-3$ mo. They had

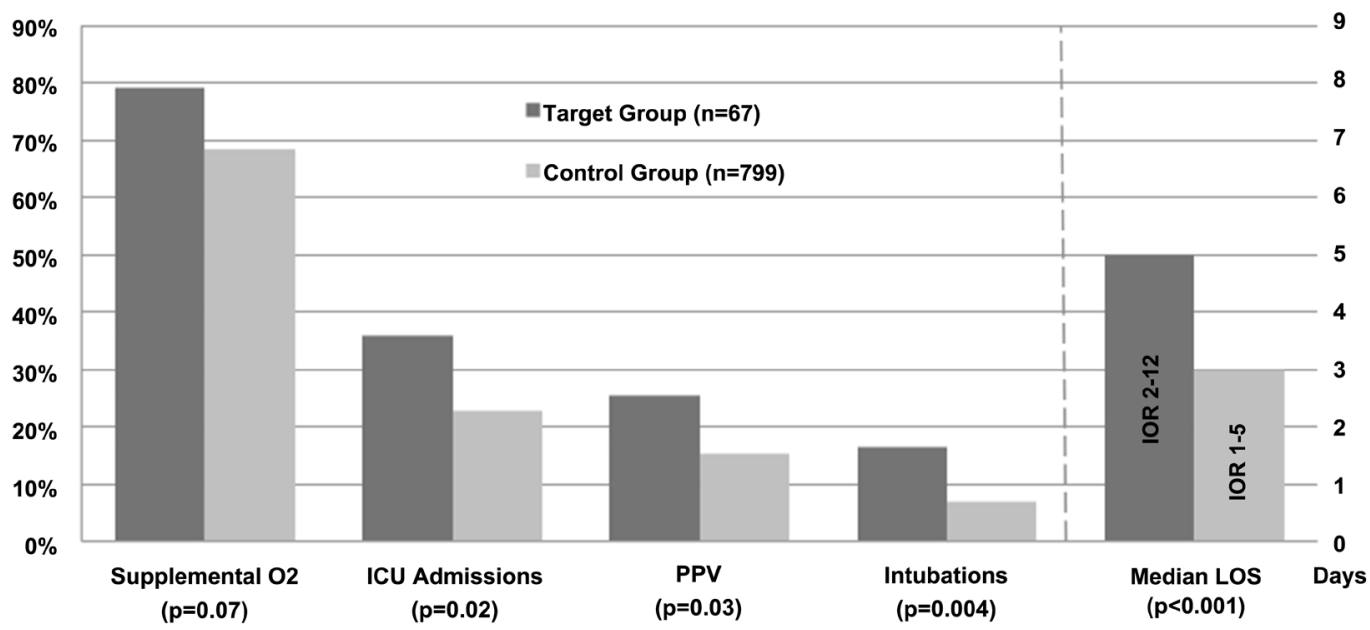

Figure 2. Disease severity of RSV hospitalizations 2012-2016: Target Group vs Control Group. Abbreviations: ICU-Intensive Care Unit; IQR - Interquartile Range; LOS_Length of stay; O2_Oxygen; PPV—Positive Pressure Ventilation; RSVH-Respiratory Syncytial Virus. 
Table 2. Disease severity among target group 2 (29 - 35 wks GA and Age $<1$ yo): Pre vs Post.

\begin{tabular}{cccccc}
\hline & \multicolumn{3}{c}{ Pre } & \multicolumn{3}{c}{ Post } \\
\hline & $\mathbf{n}$ (total 20) & $\%$ & n (total 36) & $\%$ & p-value \\
\hline Median LOS (days) & 3 & IQR & 5 & IQR & 0.20 \\
ICU Admissions & 4 & $20 \%$ & 17 & $47 \%$ & 0.05 \\
Supplemental Oxygen & 17 & $85 \%$ & 28 & $78 \%$ & 0.73 \\
PPV & 4 & $20 \%$ & 12 & $33 \%$ & 0.36 \\
Intubations & 4 & $20 \%$ & 6 & $17 \%$ & 0.73 \\
\hline
\end{tabular}

Abbreviations: GA-Gestational Age; ICU_Intensive Care Unit; IQR_Interquartile; Range; LOS-Length of stay; Pre-2012-2014; Post-2014-2016; PPV-Positive; Pressure Ventilation; wks-weeks; yo-years old.

longer LOS, greater PPV requirement, more ICU admissions, and higher intubation rates (Figure 3 ).

When comparing disease severity Pre and Post guideline change and within each age group, only patients 0 - 3 mo had significantly worse disease severity after the guidelines changed. Infants 0 - 3 mo had higher incidence of PPV, and higher rate of ICU admissions (Table 3). The median LOS was statistically higher after the guideline change for infants 0 - 3 mo but it does not appear clinically significant, Pre median 3 days (IQR 1 to 6) vs Post median 3 days (IQR 2 to 7). There were too few patients in the TG to analyze by age category.

\section{Discussion}

With the AAP's most recent update on Palivizumab prophylaxis in 2014, we found that there was no difference in the number of patients admitted for bronchiolitis who tested positive for RSV. We also found that there was no increase in the percentage of TG RSV+ subjects following the change in guidelines. However, there was a higher severity of disease among our TG who were no longer eligible for Palivizumab regardless of whether they were pre or post-2014: 1 - 2 year olds with hemodynamically significant CHD and premature infants less than 1 year of age who were born at 29 - 35 wks GA. Despite creating stricter eligibility criteria for premature infants and those with high-risk co-morbidities, our study did not observe an increase in number of RSV+ patients admitted or disease severity for either our TG or CG when compared to Pre and Post guideline changes. These findings are consistent with a study published from a tertiary care hospital involving 194 RSV hospitalized children less than 2 yo from 2012-2015. They found the rate of RSV admissions Pre (2012-2013 and 2013-2014) vs Post (2014-2015) guideline change was 5.37 per 1000 children and 5.78 per 1000 children, respectively [12]. However, this study did not focus on the specific population for which the guidelines changed.

Regardless of the modification in guidelines, our TG had significantly longer 


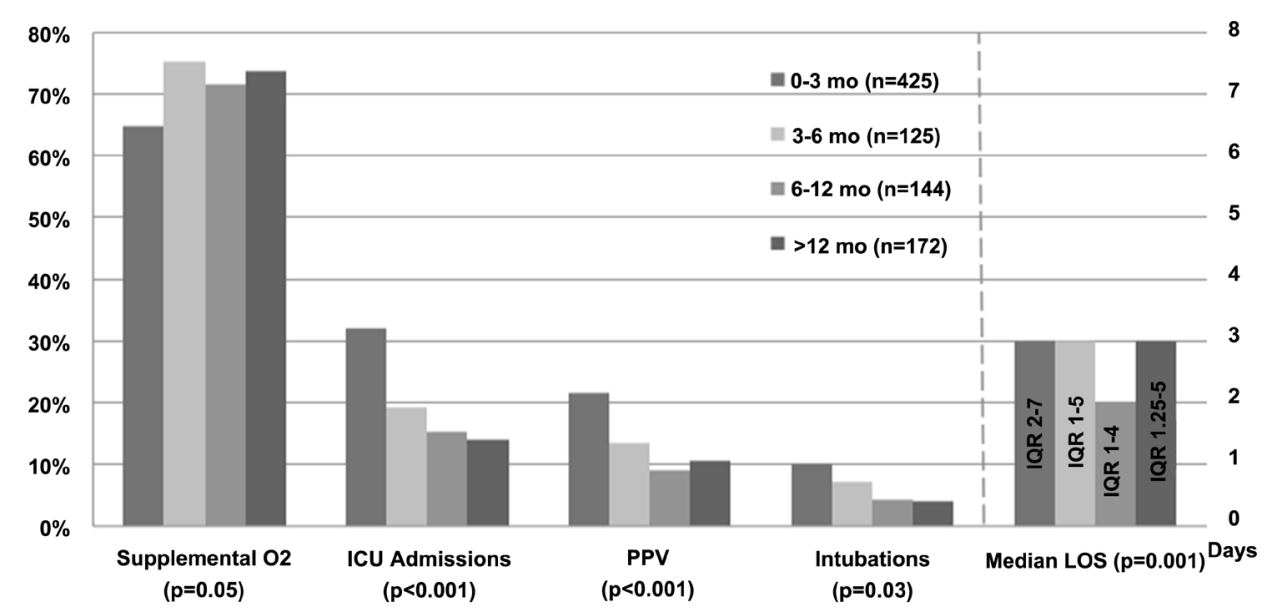

Figure 3. Disease severity of RSV hospitalizations by chronological Age 2012-2016. Abbreviations: ICU_Intensive Care Unit; IQR - Interquartile Range; LOS—Length of stay; mo-months old; O2-Oxygen; PPV—Positive Pressure Ventilation; RSV—Respiratory Syncytial Virus.

Table 3. Disease severity among age group 0 - 3 months old: Pre vs Post.

\begin{tabular}{|c|c|c|c|c|c|}
\hline & \multicolumn{2}{|c|}{ Pre } & \multicolumn{2}{|c|}{ Post } & \multirow[b]{2}{*}{ p-value } \\
\hline & $\mathrm{n}$ (total 190) & $\%$ & n (total 235) & $\%$ & \\
\hline Median LOS (days) & 3 & $\begin{array}{l}\text { IQR } \\
1-6\end{array}$ & 3 & $\begin{array}{l}\text { IQR } \\
2-7\end{array}$ & 0.04 \\
\hline ICU Admissions & 51 & $27 \%$ & 85 & $36.2 \%$ & 0.04 \\
\hline Supplemental Oxygen & 125 & $66 \%$ & 151 & $64.3 \%$ & 0.74 \\
\hline PPV & 32 & $17 \%$ & 60 & $25.5 \%$ & 0.03 \\
\hline Intubations & 19 & $10 \%$ & 24 & $10.2 \%$ & 0.94 \\
\hline
\end{tabular}

Abbreviations: ICU—Intensive Care Unit; IQR-Interquartile Range; LOS-Length Of Stay; Pre-2012-2014; Post-2014-2016; PPV—Positive Pressure Ventilation.

LOS, higher rates of ICU admissions, and required more respiratory and ventilatory support compared to CG. We also observed a strong association in disease severity with younger chronologic age and earlier GA. These younger patients experienced more complicated hospital courses in all areas that were evaluated: LOS, ICU admissions, supplemental O2, PPV, and intubations. At highest risk of severe RSV disease were patients $0-3$ mo and premature infants $<32$ wks GA.

The results from our non-industry-funded study were consistent with the industry-sponsored SENTINEL1 study, which involved 709 RSV confirmed patients from 43 geographically diverse hospitals throughout the United States. The SENTINEL1 study reported premature infants (29 - 35 wks GA) who were less than 3 mo at time of admission were at high-risk for severe RSV disease. This was supported by higher rates of RSV hospitalizations, use of invasive mechanical ventilation and ICU admissions [13]. A subsequent industry-funded study compared the rates of RSV admission for patients 29 - 34 wks GA for the initial year after the change in guidelines and found an increased rate of admission in this population of patients 0 - 3 mo [14]. Grindeland et al. showed a sim- 
ilar association with younger age and higher RSV admission rates before and after the guideline changes. That study looked at 2 seasons prior (2012-2014) and 1 season after (2014-2015) the 2014 guidelines update. They separated patients < 24 mo into three subgroups: 0 - 5 mo, $6-11$ mo, and $12-24$ mo. Among their 70 patients that were found to be RSV positive during the 2014-2015 RSV season, children 0 - 5 mo had the highest rate of admissions. They also observed admission rates trending downwards with increased chronological age [12]. Walpert et al. published a multicenter study determining the mortality and costs for patients with CHD admitted with RSV and comparing similar eras [15]. They found no change in LOS, ICU admission rates, in-hospital mortality or direct costs for those patients $13-24$ mo with CHD after the change in guidelines. Walpert et al. approached their patient selection somewhat differently. They used ICD-9 and ICD-10 codes to select for patients admitted with CHD and RSV or bronchiolitis. There was no differentiation on the type of CHD and whether or not it was hemodynamically significant. They also did not assess for actual RSV+ on viral testing and thus relied only on diagnostic coding. Regardless, they also found no differences with the changes in guidelines.

\section{Limitations}

This study has several limitations. Our study period was limited to two years before and two years after the change in guidelines. Further study with longer periods on either side would offer more insight on the effects of these changes. Given that this was a single center study, the lack of a broad demographic area makes it difficult for our data to be generalized to reflect the entire United States population. Furthermore, disease burden from RSV is not consistent and can vary by strain, geographic location, and era. We did however share similar findings with several other studies across the United States with greater generalizability. Our institution is a tertiary academic center. Thus we naturally serve a larger percentage of high-risk patients with multiple comorbidities who may benefit from Palivizumab prophylaxis. We believe this is the reason why we were able to evaluate such a large number of RSV confirmed admissions for a single center study. Of the 1283 children under 2 yo that were hospitalized with a diagnosis of bronchiolitis over this four-year study, 866 (67.5\%) were confirmed through PCR testing to have an active RSV infection. Of the remaining $417 \mathrm{pa}$ tients, 255 patients did not undergo any viral testing. Given that RSV is the major pathogen associated with bronchiolitis in this age group, it is possible that the number of patients in our study affected by this virus is much higher than what our numbers report [16]. Another limitation is restricting our initial search of potential RSV-related admissions to only diagnostic coding for bronchiolitis. Perhaps broadening our search criteria to other respiratory illnesses (e.g. pneumonitis, pneumonia, croup, and apnea) would have increased our total number of RSV confirmed admissions, which would have directly affected our final results. Having co-infection with other viruses or concurrent pneumonia may also affect overall disease burden however this was not evaluated. We also did not 
evaluate disease prevalence among different races within our study group.

\section{Conclusion}

We conclude that the changes to Palivizumab eligibility established by the AAP in 2014 did not have a significant effect on the total number of RSV+ patients admitted, the percent of RSV+ admissions who were in the target group, markers of disease severity for the overall population and for those who were no longer deemed eligible for Palivizumab between eras. However, RSV remains a significant health burden, where children of earlier GA ( $<35$ wks) and younger chronologic age $(0-3 \mathrm{mo})$ are at higher risk for a more severe disease when infected with RSV. Further studies are needed to assess for broader generalizability, larger focus on our defined target population, and potential cost analysis to deem the overall effect of the 2014 guideline changes on RSV admissions and disease severity.

\section{Conflicts of Interest}

The authors declare no conflicts of interest regarding the publication of this paper.

\section{References}

[1] Hall, C.B., Weinberg, G.A., Iwane, M.K., Blumkin, A.K., Edwards, K.M., Staat, M.A., et al. (2009) The Burden of Respiratory Syncytial Virus Infection in Young Children. New England Journal of Medicine, 360, 588-598. https://doi.org/10.1056/NEJMoa0804877

[2] Rose, E.B., Wheatley, A., Langley, G., Gerber, S. and Haynes, A. (2018) Respiratory Syncytial Virus Seasonality-United States, 2014-2017. Morbidity and Mortality Weekly Report, 67, 71-76. https://doi.org/10.15585/mmwr.mm6702a4

[3] Committee on Infectious Diseases and Bronchiolitis Guidelines Committee (2014) Updated Guidance for Palivizumab Prophylaxis among Infants and Young Children at Increased Risk of Hospitalization for Respiratory Syncytial Virus Infection. Pediatrics, 134, 415-420. https://doi.org/10.1542/peds.2014-1665

[4] Centers for Disease Control and Prevention (2013) Respiratory Syncytial Virus Activity-United States, July 2011-January 2013. Morbidity and Mortality Weekly Report, 62, 141-144.

[5] Centers for Disease Control and Prevention (2011) Respiratory Syncytial Virus-United States, July 2007-June 2011. Morbidity and Mortality Weekly Report, 60, 1203-1206.

[6] Robbie, G.J., Zhao, L., Mondick, J., Losonsky, G. and Roskos, L.K. (2012) Population Pharmacokinetics of Palivizumab, a Humanized Anti-Respiratory Syncytial Virus Monoclonal Antibody, in Adults and Children. Antimicrobial Agents and Chemotherapy, 56, 4927-4936. https://doi.org/10.1128/AAC.06446-11

[7] Megged, O. and Schlesinger, Y. (2010) Down Syndrome and Respiratory Syncytial Virus Infection. The Pediatric Infectious Disease Journal, 29, 672-673. https://doi.org/10.1097/INF.0b013e3181d7ffa5

[8] Robinson, K.A., Odelola, O.A., Saldanha, I.J. and McKoy, N.A. (2012) Palivizumab for Prophylaxis against Respiratory Syncytial Virus Infection in Children with Cys- 
tic Fibrosis. Cochrane Database of Systematic Reviews, No. 2, Article No. CD007743. https://doi.org/10.1002/14651858.CD007743.pub3

[9] Byington, C.L., Wilkes, J., Sheng, X. and Korgenski, K. (2013) Respiratory Syncytial Virus Associated Mortality in Hospitalized United States Infants and Children Less than 2 Years of Age. Pediatric Academic Societies Annual Meeting, Washington DC, 4-7 May 2013, Abstract 2915: 181.

[10] Hasegawa, K., Tsugawa, Y., Brown, D.F.M., Mansbach, J.M., Camargo, Jr., C.A. (2013) Trends in Bronchiolitis Hospitalizations in the United States, 2000-2009. Pediatrics, 132, 28-36. https://doi.org/10.1542/peds.2012-3877

[11] Hampp, C., Kauf, T.L., Saidi, A.S. and Winterstein, A.G. (2011) Cost-Effectiveness of Respiratory Syncytial Virus Prophylaxis in Various Indications. Archives of Pediatrics and Adolescent Medicine, 165, 498-505.

https://doi.org/10.1001/archpediatrics.2010.298

[12] Grindeland, C.J., Mauriello, C.T., Leedahl, D.D., Richter, L.M. and Meyer, A.C. (2016) Association between Updated Guideline-Based Palivizumab Administration and Hospitalizations for Respiratory Syncytial Virus Infections. The Pediatric Infectious Disease Journal, 35, 728-732. https://doi.org/10.1097/INF.0000000000001150

[13] Anderson, E.J., Krilov, L.R., De Vincenzo, J.P., Checchia, P.A., Halasa, N., et al. (2017) SENTINEL1: An Observational Study of Respiratory Syncytial Virus Hospitalizations among U.S. Infants Born at 29 to 35 Weeks' Gestational Age Not Receiving Immunoprophylaxis. American Journal of Perinatology, 34, 51-61. https://doi.org/10.1055/s-0036-1584147

[14] Kong, A.M., Krilov, L.R., Fergie, J., Goldstein, M., Diakun, D., et al. (2018) The 2014-2015 National Impact of the 2014 American Academy of Pediatrics Guidance for Respiratory Syncytial Virus Immunoprophylaxis on Preterm Infants Born in the United States. American Journal of Perinatology, 35, 192-200.

https://doi.org/10.1055/s-0037-1606352

[15] Walpert, A.S., Thomas, I.D., Lowe Jr., M.C. and Seckeler, M.D. (2018) RSV Prophylaxis Guideline Changes and Outcomes in Children with Congenital Heart Disease. Congenital Heart Disease, 13, 428-431. https://doi.org/10.1111/chd.12590

[16] Mansbach, J.M., Piedra, P.A., Teach, S.J., Sullivan, A.F., Forgey, T., et al. (2012) Prospective Multicenter Study of Viral Etiology and Hospital Length of Stay in Children with Severe Bronchiolitis. Archives of Pediatrics and Adolescent Medicine, 166, 700-706. https://doi.org/10.1001/archpediatrics.2011.1669 